\title{
MicroRNA-124 improves functional recovery and suppresses Bax-dependent apoptosis in rats following spinal cord injury
}

\author{
ZHONGYANG XU $^{1 *}$, KEFENG ZHANG $^{1 *}$, QIAN WANG $^{1}$ and YANPING ZHENG ${ }^{2}$ \\ ${ }^{1}$ Department of Orthopedics, Jining No. 1 People's Hospital, Jining, Shandong 272011; \\ ${ }^{2}$ Department of Orthopedics, Qilu Hospital Affiliated to Shandong University, Jinan, Shandong 250012, P.R. China
}

Received June 7, 2018; Accepted January 14, 2019

DOI: $10.3892 / \mathrm{mmr} .2019 .9904$

\begin{abstract}
Spinal cord injury (SCI) induces aberrant expression of microRNAs (miRNAs), causing various secondary injury responses, including inflammation, apoptosis and oxidative stress. However, the mechanisms underlying miRNA-mediated apoptosis have not been fully elucidated. In the present study, a rat SCI model was established and a miRNA microarray was analyzed to detect miRNA expression profiles at different times post-SCI. The Basso, Beattie and Bresnahan score, cresyl violet staining and terminal deoxynucleotidyltransferase-mediated dUTP nick end labeling staining were used to evaluate locomotor activity, lesion volume and neuronal cell apoptosis, respectively, at different time points post-SCI. It was observed that numerous miRNAs were altered at 14 days post-SCI and miR-124 was one of the most notably downregulated miRNAs. The present results demonstrated that overexpression of miR-124 by agomir-124 improves functional recovery, decreases lesion size and suppresses neuronal cell apoptosis in a rat SCI model. Luciferase reporter assay demonstrated that miR-124 inhibited apoptosis regulator BAX (Bax) expression, a key molecule in the activation of the mitochondrial apoptotic pathway, by targeting its 3'-untranslated region in BV-2 cells. Furthermore, restoration of Bax by pc-DNA-Bax inhibits the protective effect of miR-124 in $\mathrm{H}_{2} \mathrm{O}_{2}$-treated BV-2 cells. Notably, the present results demonstrated that miR-124 may block the mitochondrial apoptotic pathway by inhibiting Bax, cleaved-caspase-9 and cleaved-caspase-3 expression in rats following SCI. Collectively, the present results suggested that miR-124 may improve functional recovery and supress neuronal cell apoptosis by blocking the mitochondrial
\end{abstract}

Correspondence to: Professor Yanping Zheng, Department of Orthopedics, Qilu Hospital Affiliated to Shandong University, 107 West Wenhua Road, Jinan, Shandong 250012, P.R. China

E-mail: zhengyanping667@163.com

*Contributed equally

Key words: spinal cord injury, microRNAs, apoptosis, microRNA-124, apoptosis regulator BAX, mitochondrial apoptotic pathway apoptotic pathway in SCI rats, suggesting that miR-124 may serve as a potential therapeutic target in SCI treatment.

\section{Introduction}

Spinal cord injury (SCI) is one of the most common injuries that is identified in spine and neurosurgery departments, frequently causing permanent disabilities, including paralysis, loss of movement, sensation or autonomic control below the affected region (1). Globally, 23 cases per million occur every year (2). At present, SCI treatment is considered one of the greatest challenges for clinical practice and basic science research (3). Apoptosis is a primary difficulty in SCI treatment, which has an important role in physical and functional deficits $(4,5)$. Therefore, the development of a novel therapy is urgently required, which suppresses apoptosis in the treatment of SCI.

Apoptosis, programmed cell death, has been identified as a key process that influences the development of neuronal tissue damage following SCI (6). It was previously identified that the death receptor and mitochondrial pathways may induce apoptosis (7). The B-cell lymphoma-2 (Bcl-2) family members may mediate apoptotic signals through pro-apoptotic proteins [apoptosis regulator BAX (Bax), Bcl-2 homologous antagonist/killer and Bcl-2-associated agonist of cell death] and anti-apoptotic proteins (Bcl-2 and B-cell lymphoma-extra large) in the mitochondrial pathway (8). The collapse of the mitochondrial membrane potential is defined as a key process in the mitochondrial apoptotic pathway, which results in the translocation of cytochrome $c$ from the mitochondria into the cytosol (9). Subsequently, cytochrome $c$ together with deoxyadenosine triphosphate and apoptotic protease triggering factor- 1 in the cytosol, recruits and cleaves pro-caspase- 9 into active caspase-9 (10). In turn, activated caspase-9 cleaves effector caspases (caspase-3, -6 and -7) (11). Therefore, cytochrome $c$ release is a crucial step for activating pro-caspase- 9 in apoptotic cell death.

MicroRNAs (miRNAs) are a class of small, non-coding, single-stranded RNAs consisting of 21-23 nucleotides, which modulate post-transcriptional regulation of target genes by suppressing translation or inducing RNA degradation $(12,13)$. Previously, it was estimated that miRNAs regulate $60 \%$ of all genes in the human genome (14). A number of miRNAs were identified in the mammalian central nervous system, including 
the brain and spinal cord, where they are hypothesized to be key regulators of plasticity (15-17). Additionally, a number of microRNAs have an important role in neurodevelopment and are likely to be crucial mediators of cell differentiation into specific tissues or organs (16). Previous studies demonstrated that SCI may induce aberrant miRNA expression, which is involved in a number of secondary injury responses, including inflammation, apoptosis and oxidative stress, and regulates the expression of their target genes $(18,19)$. Recently, increasing evidence suggested that numerous miRNAs regulate apoptosis by activating the mitochondrial apoptotic pathway in various diseases (20-22). Therefore, it was hypothesized that SCI-mediated miRNAs may promote apoptosis by activating the mitochondrial apoptotic pathway.

In the present study, a rat SCI model was established and microarray analysis was conducted to determine miRNA expression profiles in spinal cord tissues. Subsequently, the role of miR-124 in SCI-induced apoptosis was examined and the underlying mechanisms in the mitochondrial apoptotic pathway were investigated.

\section{Materials and methods}

Cell culture. The immortalized murine BV-2 cell line was obtained from the Chinese Academy of Medical Science (Beijing, China) and maintained in Dulbecco's modified Eagle's medium/F12 (Gibco; Thermo Fisher Scientific, Inc., Waltham, MA, USA) containing $10 \%$ fetal bovine serum (Gibco; Thermo Fisher Scientific, Inc.), and $100 \mathrm{U} / \mathrm{ml}$ penicillin and streptomycin (Sigma-Aldrich; Merck KGaA, Darmstadt, Germany) in $25 \mathrm{~cm}^{2}$ culture flasks at $37^{\circ} \mathrm{C}$ in a humidified atmosphere with $5 \% \mathrm{CO}_{2}$.

Cell treatments. Cells were treated with different concentrations of $\mathrm{H}_{2} \mathrm{O}_{2}$ (30\% w/w solution; Sigma-Aldrich; Merck KGaA) for $10 \mathrm{~h}$ to induce cell injury. $\mathrm{H}_{2} \mathrm{O}_{2}$ was administered to the cells at 50, 100, 200 and $400 \mu \mathrm{M}$ solutions in PBS.

Experimental animals. Adult female Sprague-Dawley rats ( $\mathrm{n}=76$; age, 6 weeks; weight, 200-250 g) were obtained from the Experimental Animal Centre of Shandong University (Jinan, China). All experimental procedures were approved by the Animal Care and Use Committee of Shandong University. All animals were housed under standard laboratory conditions, in a specific-pathogen-free $\left(22 \pm 1^{\circ} \mathrm{C}\right)$ room with relative humidity of $55-65 \%$, under a normal circadian cycle $(12 \mathrm{~h}$ light/dark cycle), and had free access to food and water. All efforts were made to minimize the number of animals used and their suffering. Following adaptation to the novel environment, rats were randomly assigned to four groups; sham group, SCI group, agomir-124 group and agomir-negative control (NC) group. For the sham group ( $\mathrm{n}=6 /$ group/time), the rats underwent a T10 laminectomy without weight-drop injury. For the SCI group ( $n=6 /$ group/time), SCI was performed on the rats at the T10 spinal segment impactor. For the agomir-124 group ( $n=6 /$ group/time), SCI was performed on the rats and they were treated intrathecally with agomir-124 (1 $\mu \mathrm{l} / \mathrm{h} ; 20 \mathrm{nmol} / \mathrm{ml})$ for 3 days. For the agomir-NC group ( $n=6 /$ group/time), rats were subjected to SCI and treated intrathecally with NC agomir (1 $\mu \mathrm{l} / \mathrm{h} ; 20 \mathrm{nmol} / \mathrm{ml})$. Agomir-124 (5'-CCGUAAGUG
GCGCACGGAAU-3') and NC agomir (5'-UUCUCCGAA CGUGUCACGUTT-3') were designed and synthesized by Guangzhou RiboBio Co., Ltd. (Guangzhou, China).

Spinal cord injury model. Rats were intraperitoneally injected with $10 \%$ chloral hydrate $(350 \mathrm{mg} / \mathrm{kg})$ anaesthesia, and a laminectomy was conducted at the T9-T10 level, exposing the cord beneath without disrupting the dura. No animals exhibited peritonitis as a result of the intraperitoneal injection with $10 \%$ chloral hydrate. Subsequently, the spinous processes of T8 and T11 were clamped to stabilize the spine, and the exposed dorsal surface of the cord was subjected to weight drop injury (10 g x $25 \mathrm{~mm}$ ) using a New York University (New York, NY, USA) impactor as described previously (23). For the sham group, a T10 laminectomy without weight-drop injury was performed on the animals. All experimental protocols and post-operative animal care were approved by the Animal Care and Use Committee of Shandong University.

miRNA microarray analysis. miRNA microarray analysis was conducted to evaluate miRNA expression in the spine; rats ( $n=2 /$ group) were anesthetized at 14 days post-SCI, and a $10-\mathrm{mm}$ long segment of spinal cord, including the injury epicenter, was collected and fresh-frozen in liquid nitrogen. Total RNA was isolated from spinal cord tissues using TRIzol ${ }^{\circledR}$ reagent (Invitrogen; Thermo Fisher Scientific, Inc.) and purified by the RNeasy MinElute Cleanup kit (Qiagen $\mathrm{GmbH}$, Hilden, Germany) according to the manufacturer's protocol. Subsequent to measuring the quantity of RNA using a NanoDrop $^{\mathrm{TM}}$ ND-1000 spectrophotometer (Thermo Fisher Scientific, Inc.), the miRNAs with Hy3 were isolated using the miRCURY ${ }^{\mathrm{TM}}$ array labeling kit (Exiqon A/S, Vedbaek, Denmark) and hybridized on a miRCURY TM LNA Array (version 18.0; Exiqon A/S). The Axon GenePix 4000B microarray scanner (Molecular Devices, LLC, Sunnyvale, CA, USA) was used to scan the slides. The scanned images were analyzed with the GenePix Pro6.0 program (Molecular Devices, LLC). The miRNAs with intensities $\geq 50$ in all samples were used to calculate a normalization factor. Expressed data were normalized by median normalization. Subsequently, the miRNAs were measured by Volcano Plot filtering. Finally, hierarchical clustering was used to determine the differences in the miRNA expression profiles using MultiExperiment Viewer software (version 4.6; The Institute for Genomic Research, Rockville, MA, USA) (24).

Reverse transcription-quantitative polymerase chain reaction $(R T-q P C R)$. Total RNA from spinal cord segments containing the injury epicenter was isolated using TRIzol ${ }^{\circledR}$ (Invitrogen; Thermo Fisher Scientific, Inc.), according to the manufacturer's protocol. RT was performed using the TaqMan ${ }^{\mathrm{TM}}$ MicroRNA Reverse Transcription kit (Applied Biosystems; Thermo Fisher Scientific, Inc.), according to the manufacturer's instructions. PCR was performed using the TaqMan ${ }^{\mathrm{TM}}$ MicroRNA Assay kit (Applied Biosystems; Thermo Fisher Scientific, Inc.) on an Applied Biosystems 7500 Fast Real-Time PCR system (Thermo Fisher Scientific, Inc.). The thermocycling conditions were as follows: $50^{\circ} \mathrm{C}$ for $2 \mathrm{~min}$ and $95^{\circ} \mathrm{C}$ for $10 \mathrm{~min}$, followed by 40 cycles of $95^{\circ} \mathrm{C}$ for $15 \mathrm{sec}$ and $60^{\circ} \mathrm{C}$ for $10 \mathrm{~min}$. The following 
primer sequences were used: miR-124, 5'-TAAGGCACGCGG TGAATGCC-3' (forward) and 5'-AATGATACGGCGACC ACCGAACACTCTTTCCCTACACGACG-3' (reverse); Bax, 5'-GGCTGGACACTGGACTTCCT-3' (forward) and 5'-GGT GAGGACTCCAGCCACAA-3' (reverse); and U6, 5'-CTC GCTTCGGCAGCACA-3' (forward) and 5'-AACGCTTCA CGAATTTGCGT-3' (reverse). The relative expression of miRNAs was normalized to U6. Data were analyzed using the $2^{-\Delta \Delta \mathrm{Cq}}$ method, as previously described (25). All reactions were performed in triplicate.

Behavior assessment. The Basso, Beattie and Bresnahan (BBB) score was used to evaluate the locomotor activity at $1,7,14$ and 28 days post-SCI (26), which measured locomotor ability for $4 \mathrm{~min}$. Behavioral analyses were performed by trained investigators, who were blind to the experimental conditions. To test hind limb locomotor function, open-field locomotion was assessed using the BBB locomotion scale, as previously described $(23,26)$. The final score from two investigators was averaged for each rat.

Assessment of lesion volume. To measure the lesion volume following SCI or treatment with agomir-124, the rats were intraperitoneally injected with $10 \%$ chloral hydrate (350 mg/kg) for anaesthesia. No animals experienced peritonitis as a result of the intraperitoneal injection of $10 \%$ chloral hydrate. They were subsequently transcardially perfused with $0.9 \% \mathrm{NaCl}\left(250 \mathrm{ml} ; 4^{\circ} \mathrm{C}\right)$ followed by $4 \%$ paraformaldehyde (PFA; $500 \mathrm{ml}$ ) in $0.1 \mathrm{M} \mathrm{PBS}(\mathrm{pH} 7.4)$ at $4^{\circ} \mathrm{C}$ for $30 \mathrm{~min}$. A $1 \mathrm{~cm}$ segment of spinal cord containing the injury epicenter was removed and post-fixed in the same fixative at $4^{\circ} \mathrm{C}$ for $24 \mathrm{~h}$. The tissue blocks were embedded in paraffin following fixation. Transverse sections (10 $\mu \mathrm{m}$ thickness) were taken through the width of the spinal lesion site, and mounted onto Superfrost Plus Slides (Thermo Fisher Scientific, Inc.). Samples (every 40th section of the lesion site) were stained with $0.5 \%$ cresyl-violet acetate for $1 \mathrm{~h}$ at room temperature and imaged using an Olympus BH-2 microscope (magnification, x200; Olympus Corporation, Tokyo, Japan). The lesion area and spared tissue area were outlined and quantified using Image-Pro Plus 6.0 (Media Cybernetics, Inc., Rockville, MD, USA) software. Spared tissue was reported as the remaining areas, where normal spinal cord structure was preserved. The section with the lowest percentage of spared tissue was defined as the injury epicenter. Transverse sections, with intervals of $400 \mu \mathrm{m}$ rostral and caudal to this lesion epicenter, were analyzed up to a distance of $1,600 \mu \mathrm{m}$ away from the lesion epicenter for percentage tissue sparing.

Terminal deoxynucleotidyl-transferase-mediated dUTP nick end labeling (TUNEL) staining. To detect apoptosis, serial spinal cord sections (10 $\mu \mathrm{m}$ thickness), obtained in the above experimental procedure, were further subjected to TUNEL staining. The TUNEL Apoptosis Assay kit (cat. no. 11684817910; Roche Diagnostics GmbH, Mannheim, Germany), was used to detect apoptotic cells in spinal cord sections, according to the manufacturer's protocol. The tissue sections were immersed in the TUNEL reaction mixture for $1 \mathrm{~h}$ at $37^{\circ} \mathrm{C}$. Subsequently, nuclei were stained with $1 \mu \mathrm{g} / \mathrm{ml}$ DAPI at room temperature for $10 \mathrm{~min}$. Sections were mounted in Fluoromount ${ }^{\mathrm{TM}}$ aqueous mounting medium (Sigma-Aldrich; Merck KGaA). Quantification was conducted by counting the number of positive cells in 10 randomly chosen fields within each slide with a Leica CM 1850 fluorescent microscope (magnification, $\mathrm{x} 200$ ). Apoptosis was determined by measuring the apoptotic cells and the total cells.

Luciferase reporter assay. The potential binding site between Bax and miR-124 was searched in TargetScan (http://www.targetscan.org). The miR-124 mimics/inhibitor and corresponding $\mathrm{NC}$ were designed and synthesized by Guangzhou RiboBio Co., Ltd. (Guangzhou, China). The fragment of the 3'-untranslated region (UTR) of Bax [wild-type (wt) or mutant (mut)] was amplified and cloned into the pMIR-REPORT luciferase vector (Ambion; Thermo Fisher Scientific, Inc.). Site-directed mutagenesis of the Bax 3'-UTR at the putative miR-124 binding site was performed using a QuikChange II Site-Directed Mutagenesis kit (Agilent Technologies, Inc., Santa Clara, CA, USA). Subsequently, BV-2 cells at a density of $2 \times 10^{5}$ cells/well were seeded into 24 -well plates and co-transfected with $0.8 \mu \mathrm{g}$ pMIR-Bax-3'-UTR or pMIR-Bax-mut-3'-UTR, $50 \mathrm{nM}$ miR-124 mimics/inhibitor or corresponding NC using Lipofectamine ${ }^{\circledR} 2000$ reagent (Invitrogen; Thermo Fisher Scientific, Inc.). Renilla luciferase was used to normalize the cell number $48 \mathrm{~h}$ after transfection. Luciferase activity was measured using the Dual-Light luminescent reporter gene assay (Applied Biosystems; Thermo Fisher Scientific, Inc.). Each assay was repeated three times.

Western blot analysis. Segments of spinal cord $(1 \mathrm{~cm})$ were isolated using the lesion site as the epicenter, and protein was extracted from spinal cord tissues or BV-2 cells using ice cold radioimmunoprecipitation assay buffer (Roche Diagnostics $\mathrm{GmbH}$ ) as previously described (19). Samples were sonicated using a W-385 sonicator (Qsonica LLC, Newtown, CT, USA) at room temperature, $30 \mathrm{sec}$ intervals and power level six $\left(20 \mathrm{kHz}\right.$ ) for $15 \mathrm{~min}$, then centrifuged at $20,000 \mathrm{x} \mathrm{g}$ at $4^{\circ} \mathrm{C}$ for $30 \mathrm{~min}$. Supernatants were collected and stored at $-80^{\circ} \mathrm{C}$. The protein expression level of the supernatant was determined using a bicinchoninic acid (BCA) assay (Beyotime Institute of Biotechnology, Haimen, China). Samples (30 $\mu \mathrm{g}$ protein) were electrophoresed onto 12\% SDS/PAGE (Sigma-Aldrich; Merck KGaA), and transferred to polyvinylidene difluoride membranes (EMD Millipore, Billerica, MA, USA). Subsequent to blocking with $5 \%$ non-fat milk at $4^{\circ} \mathrm{C}$ overnight, the membranes were incubated at $4^{\circ} \mathrm{C}$ overnight with primary antibodies against Bax (1:1,000; cat. no. 2772; Cell Signaling Technology, Inc., Danvers, MA, USA), Bcl-2 (1:1,000; cat. no. sc-492; Santa Cruz Biotechnology, Inc., Dallas, TX, USA), cleaved-caspase-9 (1:1,000; cat. no. 9509; Cell Signaling Technology, Inc.), pro-caspase-9 (1:1,000; cat. no. sc-56073; Santa Cruz Biotechnology, Inc.), cleaved-caspase-3 (1:1,000; cat.no.9661; Cell Signaling Technology,Inc.) and pro-caspase-3 (1:1,000; cat. no. 9662s; Cell Signaling Technology, Inc.). $\beta$-actin (1:1,000; cat. no. A1978; Sigma-Aldrich; Merck KGaA) was used as an internal control. The membrane was then incubated at $4^{\circ} \mathrm{C}$ for $90 \mathrm{~min}$ with anti-mouse IgG horseradish peroxidase-conjugated secondary antibody (1:200; cat. no. 7076; Cell Signaling Technology, Inc.). Protein expression was detected using Amersham ${ }^{\mathrm{TM}} \mathrm{ECL}^{\mathrm{TM}}$ Western Blotting 
Detection Reagents (cat. no. RPN2106; GE Healthcare Life Sciences, Little Chalfont, UK). The protein bands were quantified using a PhosphorImager and ImageQuant 5.2 software (GE Healthcare Life Sciences).

Immunohistochemistry staining. The spinal cords were intracardially perfused with $0.9 \% \mathrm{NaCl}$ followed by ice-cold $4 \%$ PFA in $0.1 \mathrm{M}$ PBS (pH 7.4) at $4^{\circ} \mathrm{C}$ for $30 \mathrm{~min}$. A $10 \mathrm{~mm}$ segment of spinal cord encompassing the injury site was subsequently harvested. Following fixation, the tissue blocks were embedded in paraffin, and sectioned at $5 \mu \mathrm{m}$ thickness. Paraffin-embedded sections were deparaffinized with xylene at $50^{\circ} \mathrm{C}$ for $3 \mathrm{~min}$ and hydrated through a graded alcohol series $(100,95,80,70$ and 40\%). Epitope unmasking was performed by microwave irradiation in $10 \mathrm{mM}$ citrate buffer (pH 6.0) twice for $5 \mathrm{~min}$ at $800 \mathrm{~W}$ prior to cooling for $30 \mathrm{~min}$. Subsequently, endogenous peroxidase was inactivated by incubation in $3 \% \mathrm{H}_{2} \mathrm{O}_{2}$ for $15 \mathrm{~min}$ at room temperature. Subsequent to rinsing with $0.01 \mathrm{M}$ PBS, the sections were blocked with $10 \%$ fetal bovine serum (cat. no. F4135; Sigma-Aldrich; Merck KGaA) in PBS at room temperature for $30 \mathrm{~min}$ and incubated overnight at $4^{\circ} \mathrm{C}$ with primary anti-cleaved-caspase- 3 antibodies (1:100; cat. no. 9661; Cell Signaling Technology, Inc.). Subsequently, the sections were incubated with anti-mouse IgG horseradish peroxidase-conjugated secondary antibodies (1:100; cat. no. 7076; Cell Signaling Technology, Inc.) at room temperature for $30 \mathrm{~min}$. Finally, the immunoreactivity was visualized by staining with 3,3'-diaminobenzidine at room temperature for $3 \mathrm{~min}$, covered with a coverslip. Images were photographed using an Olympus BX51 light microscope (magnification, x200; Olympus Corporation). Aperio ImageScope version 9 was used to quantify immunohistochemistry staining (Leica Biosystems Nussloch GmbH, Nussloch, Germany).

Apoptosis analysis by flow cytometry. BV-2 cells $\left(1 \times 10^{6}\right)$ were harvested and washed in ice-cold PBS, and fixed in 70\% ice-cold ethanol in PBS for $30 \mathrm{~min}$. To measure apoptosis, the fluorescein isothiocyanate (FITC) Annexin V Apoptosis Detection kit I (BD Bioscience, Franklin Lakes, NJ, USA) was used according to the manufacturer's instructions. Cells were washed twice in PBS, resuspended in Annexin V binding buffer, and incubated with $5 \mu \mathrm{l}$ Annexin V-FITC and $1 \mu \mathrm{l}$ propidium iodide (PI). The stained cells were analyzed using a flow cytometer (EPICS XL-MCL FACScan; BD Biosciences, Franklin Lakes, NJ, USA). The MultiCycle Software version 5.0 (Phoenix Flow Systems, San Diego, CA, USA) for Windows 7 (Microsoft Corporation, Redmond, WA, USA) was used to analyze the experimental data.

Caspase- 3 activity. Caspase- 3 activity was determined using a colorimetric activity assay kit, according to the manufacturer's protocol (BioVison, Inc., Milpitas, CA, USA). BV-2 cells were harvested by centrifugation at $1,000 \mathrm{x}$ g for $10 \mathrm{~min}$ at $4^{\circ} \mathrm{C}$ and incubated in lysis buffer on ice for $15 \mathrm{~min}$. Subsequently, the lysate was centrifuged at $20,000 \times \mathrm{g}$ for $15 \mathrm{~min}$ at $4^{\circ} \mathrm{C}$, the protein concentration was measured using the BCA Protein Assay kit (Beyotime Institute of Biotechnology) according to the manufacturer's protocol. The lysates $(10 \mu \mathrm{l})$ were incubated with $10 \mu \mathrm{l} 0.2 \mathrm{mM}$ Ac-DEVD-pNA in $80 \mu \mathrm{l}$ reaction buffer at $37^{\circ} \mathrm{C}$ for $2 \mathrm{~h}$. The samples were measured with a microplate reader (Model 680; Bio-Rad Laboratories, Inc., Hercules, CA, USA) at an absorbance of $405 \mathrm{~nm}$.

Statistical analysis. All statistical analyses were performed using SPSS software (version 18.0; SPSS, Inc., Chicago, IL, USA) or GraphPad Prism (version 6.0; GraphPad Software, Inc., La Jolla, CA, USA). Experiments were conducted in triplicate and data are presented as the mean \pm standard deviation. The correlation between Bax and miR-124 expression was analyzed using Spearman's rank correlation coefficient. Differences among multiple groups were analyzed by one-way analysis of variance with Tukey's post hoc test, and differences between two groups were analyzed by Student's t-test. $\mathrm{P}<0.05$ was used to indicate a statistically significant difference.

\section{Results}

miRNA aberrant expression in rats following SCI. To examine the potential involvement of miRNA in SCI, a rat model of SCI was established and microarray analysis was performed to determine miRNA expression levels in spinal cord tissues. It was observed that a large number of miRNAs were altered at 14 days post-SCI and that miR-124 was one of the miRNAs most significantly downregulated compared with the sham group (Fig. 1A). A previous study observed that miR-124 is relatively abundant in the spinal cord neurons, brain and retina, and serves a critical role in neural development and differentiation (27). Therefore, RT-qPCR was performed to further verify the miR-124 expression level in spinal cord tissues at 1, 7, 14 and 28 days post-SCI and it was observed that it was additionally significantly downregulated in the SCI group compared with the sham group between 7 and 28 days $(\mathrm{P}<0.05$; Fig. 1B). These results suggested that SCI results in miRNA aberrant expression in spinal cord tissues and miR-124 may regulate the pathogenesis in rats with SCI.

Overexpression of miR-124 improves functional recovery and inhibits apoptosis following SCI. To investigate the role of miR-124 in rats with SCI, a rat SCI model was established and treated intrathecally with agomir-124. The overexpression effect of agomir-124 in the spinal cord was assessed using RT-qPCR. As demonstrated in Fig. 2A, the relative expression of miR-124 was significantly upregulated at 7-28 days compared with the agomir-NC $(\mathrm{P}<0.05)$ and was maximal at 14 days. Subsequently, the BBB rating scale was used to evaluate motor function in the rat SCI model following treatment intrathecally with agomir-124. The results demonstrated that overexpression of miR-124 in the SCI + agomir-124 group significantly improved motor function from 7 days compared with the SCI group ( $\mathrm{P}<0.05$; Fig. $2 \mathrm{~B})$. Cresyl violet staining demonstrated that the spared tissue in SCI + agomir-124 group was significantly increased compared with the SCI group ( $\mathrm{P}<0.05$; Fig. $2 \mathrm{C})$, suggesting agomir-124 reduces lesion volume in spinal cord tissues following SCI. It was additionally investigated whether miR-124 modulates apoptosis-associated protein (cleaved-caspase-3) expression using immunohistochemistry staining in spinal cord tissues following SCI. As demonstrated in Fig. 2D, SCI resulted in a significant upregulation of cleaved-caspase-3 in spinal cord tissues compared 
with the shame group; whereas, agomir-124 significantly inhibited the cleaved-caspase-3 expression ( $\mathrm{P}<0.01$; Fig. 2D). Furthermore, neuronal cell apoptosis was analyzed using TUNEL staining. It was identified that the TUNEL-positive cells were significantly increased in the SCI group compared with the sham group $(\mathrm{P}<0.01)$; however, agomir-124 significantly decreased the number of TUNEL-positive cells in the SCI + agomir-124 group compared with the SCI group $(\mathrm{P}<0.01$; Fig. 2E). These results suggested that agomir-124 improves functional recovery, reduces lesion volume and suppresses apoptosis in rats following SCI.

miR-124 suppresses Bax expression by targeting its 3'-UTR in $B V-2$ cells. A previous study suggested that miR-124 exerts protective effects against neuron apoptosis in rats with thyroid hypofunction via downregulation of pro-apoptosis proteins Bax and caspase-3 (28). Therefore, it was hypothesized that miR-124 inhibits neuronal cell apoptosis in rats with SCI via downregulation of Bax. Bioinformatics analysis was conducted to predict the putative targets of miR-124, and it was observed that Bax may be a target gene of miR-124 with the target site located in the 3'-UTR (Fig. 3A). To validate this bioinformatics predication, the wild-type (wt) or mutant (mut) type of Bax-3'-UTR was constructed, which was inserted into the firefly luciferase expressing the vector pMIR-REPORT. To investigate the pathologic factors following SCI, the BV-2 cell line was used as it is reported to share various characteristics with primary microglia (29). The reporter plasmids were co-transfected with either miR-124 mimics/inhibitor or mimics/inhibitor NC in BV-2 cells, and the luciferase activity was measured. Compared with the mimics NC, the miR-124 mimics significantly inhibited the luciferase activity in the presence of the wt 3'-UTR; whereas, the miR-124 inhibitor significantly increased the luciferase activity compared with the inhibitor NC ( $\mathrm{P}<0.01$; Fig. 3B). Additionally, miR-124 did not suppress the luciferase activity of the reporter vector containing 3'-UTR of Bax with mutations in the miR-124-binding site (Fig. 3B). To further verify that Bax is negatively regulated by miR-124, western blotting and RT-qPCR analysis was performed to detect the protein and mRNA expression level for Bax, respectively. It was observed that overexpression of miR-124 decreased the expression of Bax at the mRNA and protein expression level in BV-2 cells (Fig. 3C and D). Conversely, downregulation of miR-124 increased the Bax expression at the mRNA and protein expression level (Fig. 3C and D). Furthermore, RT-qPCR analysis was used to determine the Bax mRNA expression level in spinal cord tissues $(\mathrm{n}=8)$. It was demonstrated that the Bax mRNA expression level was significantly increased in the SCI group compared with the sham group $(\mathrm{P}<0.01 ;$ Fig. 3E). The correlation analysis revealed a strong negative correlation between Bax and miR-124 expression in spinal cord tissues ( $\mathrm{r}=-0.9043$; $\mathrm{P}<0.01$; Fig. 3F). Collectively, these results suggested that miR-124 suppressed Bax expression by directly targeting its 3'-UTR in BV-2 cells, suggesting that Bax may be a target of miR-124 in spinal cord tissues.

Overexpression of Bax inhibits the protective effect of miR-124 on $\mathrm{H}_{2} \mathrm{O}_{2}$-treated $\mathrm{BV}-2$ cells. A previous study demonstrated that reactive oxygen species have key roles in SCI as they may

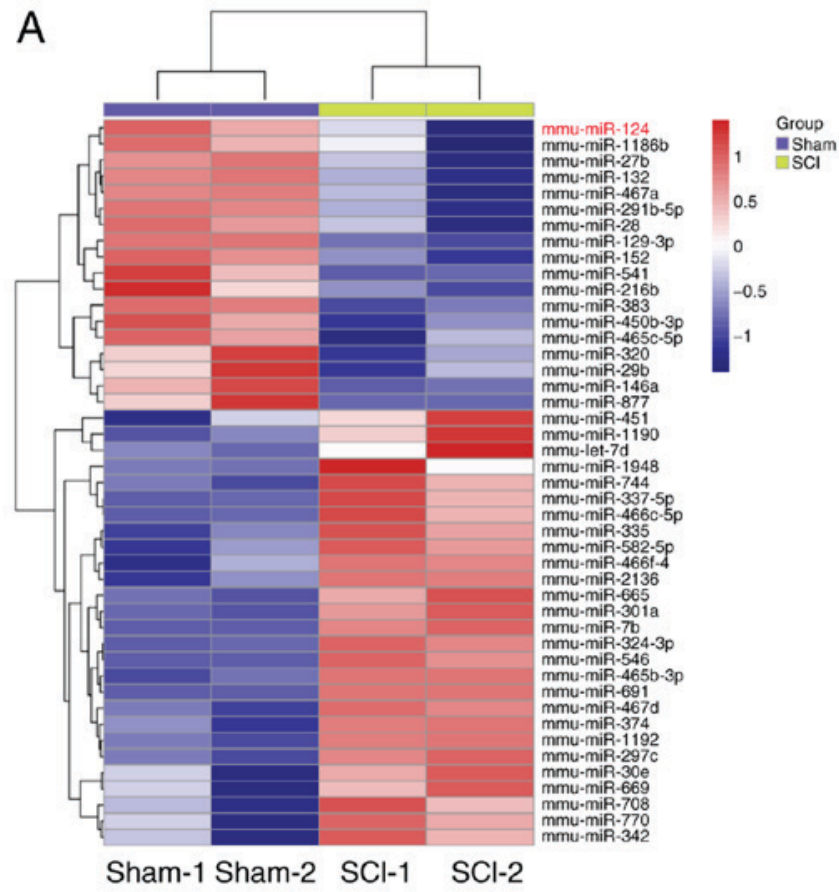

B

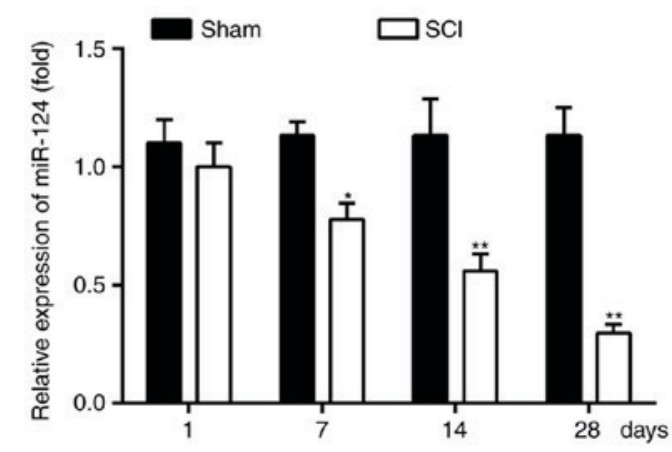

Figure 1. Cluster analysis of miRNA expression in rats with SCI. (A) Heat map presents significant expressional alterations of miRNA expression levels in rats at 14 days post-SCI ( $\mathrm{n}=2$ /group). Blue indicates downregulation and red indicates upregulation. (B) Reverse transcription-quantitative polymerase chain reaction was used to determine the miR-124 expression level in spinal cord tissues at 1, 7, 14 and 28 days post-SCI ( $=6 /$ group/time). Data are presented as the mean \pm standard deviation of three individual experiments. ${ }^{*} \mathrm{P}<0.05$, ${ }^{* *} \mathrm{P}<0.01$ vs. respective sham. $\mathrm{miRNA} / \mathrm{miR}$, microRNA; SCI, spinal cord injury.

activate various pathways of apoptosis, and $\mathrm{H}_{2} \mathrm{O}_{2}$-treated $\mathrm{BV}-2$ cells widely serve as a cellular model of SCI to examine the pathologic factors following SCI (30). In the present study, murine BV-2 cells were treated with $\mathrm{H}_{2} \mathrm{O}_{2}(50-400 \mu \mathrm{M})$ for $10 \mathrm{~h}$, and the miR-124 expression was measured using RT-qPCR analysis. As demonstrated in Fig. 4A, treatment with $\mathrm{H}_{2} \mathrm{O}_{2}$ results in the significant downregulation of miR-124 in BV-2 cells, and miR-124 downregulation was dose-dependent at $\mathrm{H}_{2} \mathrm{O}_{2}$ concentrations of $50-200 \mu \mathrm{M}(\mathrm{P}<0.05)$. Furthermore, RT-qPCR and western blot analysis was performed to evaluate the overexpression efficiency of miR-124 or Bax, respectively. The results demonstrated that miR-124 and Bax was upregulated in BV-2 cells treated with miR-124 mimics and pc-DNA-Bax, respectively (Fig. 4B and C). In addition, it was observed that overexpression of miR-124 decreased the Bax protein expression level in $\mathrm{H}_{2} \mathrm{O}_{2}$-treated $\mathrm{BV}-2$ cells; whereas, the restoration of Bax by pc-DNA-Bax inhibited 
A

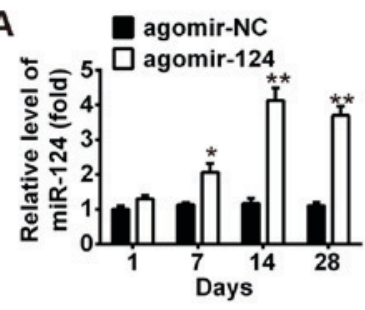

B

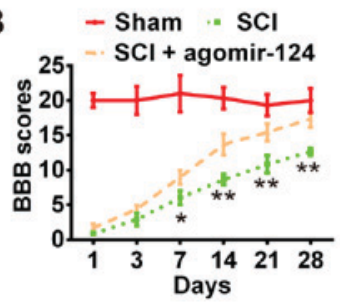

C

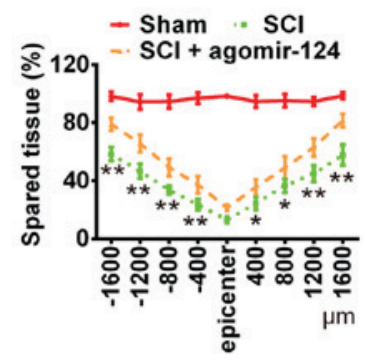

D

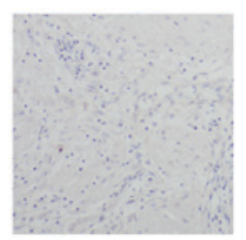

Sham

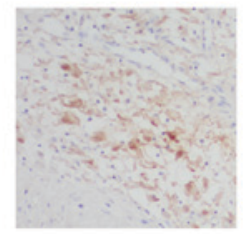

scl

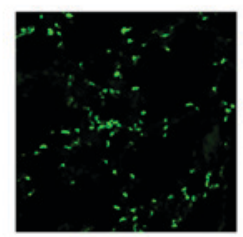

SCl

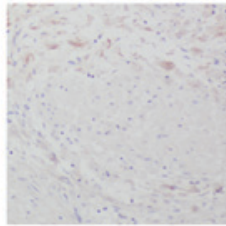

$\mathrm{SCl}+$ agomir-124

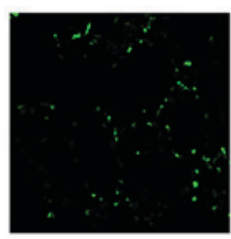

$\mathrm{SCl}+$ agomir-124
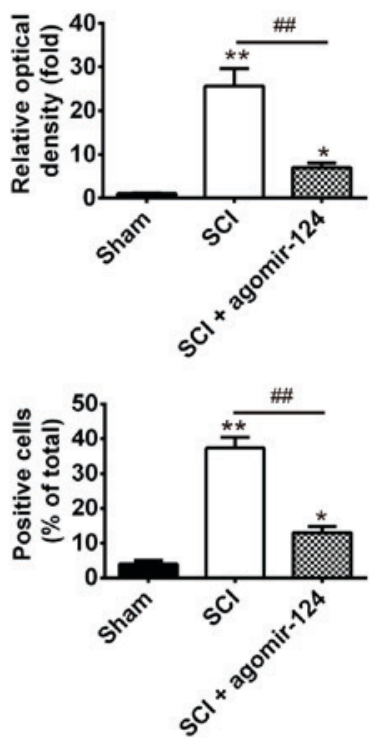

Figure 2. Agomir-124 alleviates SCI. (A) Relative expression level of miR-124 was detected by reverse transcription-quantitative polymerase chain reaction at $1,7,14$ and 28 days in rats SCI model following intrathecal treatment with agomir- $124(\mathrm{n}=6 /$ group $/$ time $) .{ }^{*} \mathrm{P}<0.05,{ }^{* *} \mathrm{P}<0.01$ vs. respective agomir-NC. (B) BBB score was used to evaluate locomotor activity at 1, 3, 7, 14 and 28 days post-SCI following agomir-124 treatment (n=6/group/time). (C) Lesion analysis at the rostral (-) and caudal (+) margins from the lesion epicenter was performed using cresyl violet staining 14 days after the SCI model was established ( $\mathrm{n}=6$ /group/time). ${ }^{*} \mathrm{P}<0.05,{ }^{* *} \mathrm{P}<0.01$ vs. SCI + agomir-124 group. (D) Immunohistochemistry staining was used to detect cleaved-caspase-3 expression in spinal cord tissues following SCI (magnification, $\mathrm{x} 200$ ). ${ }^{*} \mathrm{P}<0.05,{ }^{* *} \mathrm{P}<0.01 \mathrm{vs}$. sham group; ${ }^{\# \#} \mathrm{P}<0.01$. (E) Terminal deoxynucleotidyl transferase dUTP nick end labeling staining was used to analyze neuronal apoptosis at 14 days post-SCI (n=6/group/time) (magnification, $\mathrm{x} 200)$. Data are presented as the mean \pm standard deviation of three individual experiments. SCI, spinal cord injury; miR, microRNA; NC, negative control; BBB, Basso, Beattie and Bresnahan.

the effect of miR-124-reduced Bax on $\mathrm{H}_{2} \mathrm{O}_{2}$-treated $\mathrm{BV}-2$ cells (Fig. 4D). Notably, the present results demonstrated that overexpression of miR-124 significantly decreased the portion of apoptotic cells in $\mathrm{H}_{2} \mathrm{O}_{2}$-treated $\mathrm{BV}-2$ cells compared with the control; however, the protective effect of miR-124 on BV-2 cells was significantly decreased with Bax overexpression $(\mathrm{P}<0.01$; Fig. 4E). Furthermore, upregulation of Bax inhibited the effect of miR-124-inhibited caspase-3 activity in $\mathrm{H}_{2} \mathrm{O}_{2}$-treated BV-2 cells $(\mathrm{P}<0.01$; Fig. 4F). Taken together, these results suggested that miR-124 suppresses cell apoptosis by inhibiting $\mathrm{Bax}$ in $\mathrm{H}_{2} \mathrm{O}_{2}$-treated $\mathrm{BV}-2$ cells.

Overexpression ofmiR-124blocks the mitochondrial apoptotic pathway. Bax, is a $\mathrm{Bcl}-2$ family protein (31), which has been identified to possess a pro-apoptotic effect and causes the release of cytochrome $c(32,33)$. In the apoptotic process, the upregulation of Bax may promote apoptosis by inhibiting the anti-apoptotic protein Bcl-2 (34). The mitochondrial apoptotic pathway and death receptor apoptotic pathway are two important apoptotic pathways $(35,36)$. Bax is a key molecule in the regulation of the mitochondrial apoptotic pathway, which is able to form homodimers on the mitochondrial membrane and open the permeability transition pore on the mitochondrial membrane to promote the release of cytochrome $c$ from mitochondria into the cytoplasm (37). In the mitochondrial apoptotic pathway, cytochrome $c$ may active the caspase-9-mediated cascade amplification reaction, which in turn processes pro-caspase-3 to generate active caspase-3 (38). To investigate whether miR-124 may regulate the mitochondrial apoptotic pathway by suppressing apoptotic-associated protein expression in rats following SCI, western blotting was used to detect the Bax, Bcl-2, cleaved-caspase-9, pro-caspase-9, cleaved-caspase-3 and pro-caspase-3 expression levels in spinal cord tissues. It was observed that Bax, cleaved-caspase- 9 and cleaved-caspase- 3 were significantly upregulated, and $\mathrm{Bcl}-2$, pro-caspase- 9 and pro-caspase-3 were significantly downregulated in the SCI + agomir-NC group compared with the sham group. However, overexpression of miR-124 significantly decreased the Bax, cleaved-caspase- 9 and cleaved-caspase-3 expression levels and significantly increased the Bcl-2, pro-caspase- 9 and pro-caspase- 3 expression levels in the $\mathrm{SCI}+$ agomir-124 group compared with the SCI + agomir-NC group $(\mathrm{P}<0.01$; Fig. 5). These results suggested that overexpression of miR-124 may block the mitochondrial apoptotic pathway by suppressing Bax expression in rats following SCI. 
A

$\begin{array}{lr}\text { mmu-miR-124 3'-ccguaaguggcgcACGGAAu-5' } \\ \text { Bax wt } & \text { 5'-ggccucccacUGCCUUg-3' } \\ \text { Bax mut } & \text { 5'-ggccucccac GAGUAC g-3' }\end{array}$
B

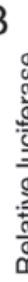

C

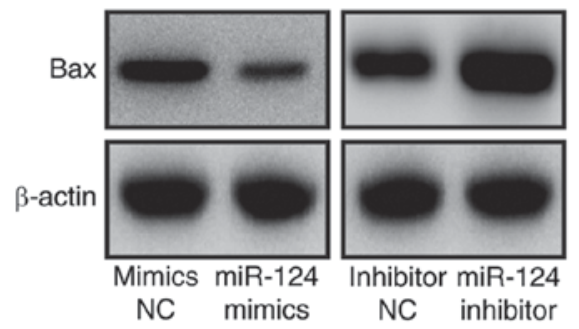

E

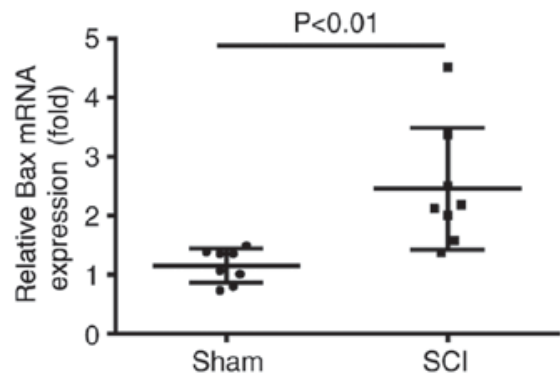

D
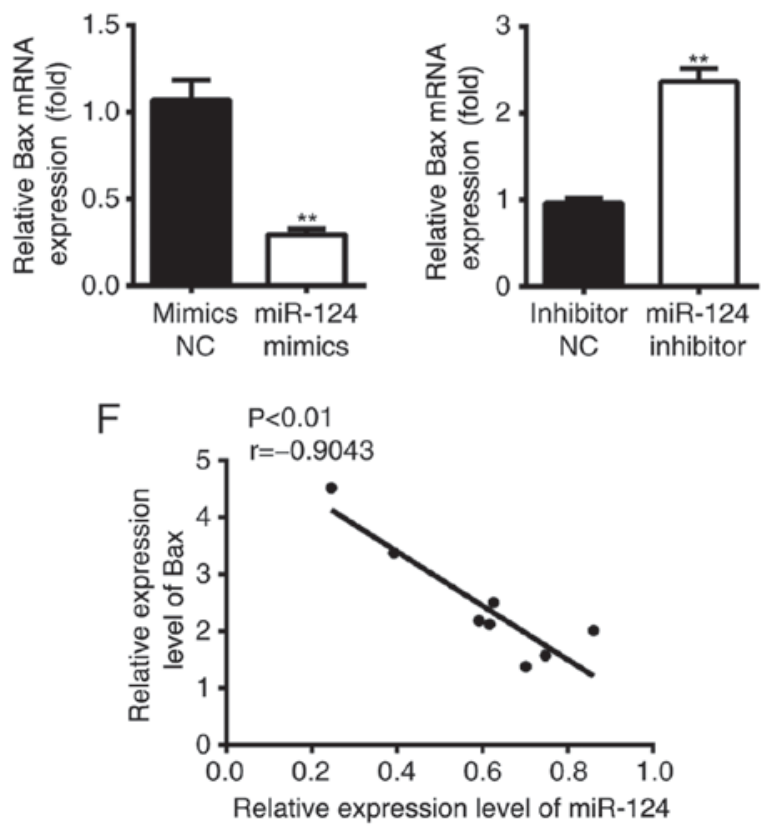

Figure 3. Bax is a direct target of miR-124 in BV-2 cells. (A) Putative miR-124 binding sites on 3'-UTR of Bax mRNA were predicted using TargetScan. (B) Relative luciferase activity of Bax wt or mut 3'-UTR in BV-2 cells following transfection with the miR-124 mimics/inhibitor or corresponding NC. ${ }^{* *} \mathrm{P}<0.01 ;{ }^{\# \#} \mathrm{P}<0.01$. BV-2 cells were transfected with miR-124 mimics/inhibitor or corresponding NC, the (C) protein and (D) mRNA expression levels of Bax were determined using western blotting and RT-qPCR, respectively. $\beta$-actin was used as an internal control. ${ }^{* *} \mathrm{P}<0.01$ vs. respective NC. (E) RT-qPCR was used to determine the mRNA expression of Bax in spinal cord tissues at 14 days post-SCI. $n=8$. (F) Negative correlation between Bax and miR-124 expression levels in spinal cord tissues at 14 days post-SCI ( $\mathrm{r}=-0.9043 ; \mathrm{P}<0.01)$. Data are presented as the mean \pm standard deviation of three independent experiments. Bax, apoptosis regulator BAX; miR, microRNA; 3'-UTR, 3'-untranslated region; NC, negative control; RT-qPCR, reverse transcription-quantitative polymerase chain reaction; wt, wild-type; mut, mutant.

\section{Discussion}

SCI induces widespread molecular and biochemical alterations, which are characterized by the production of free radicals, inflammatory activation, axonal plasticity and neuronal cell death $(39,40)$. Previously, certain studies have clarified that SCI may induce miRNA aberrant expression and the dysregulated miRNAs may influence SCI pathophysiology and functional outcome $(18,41)$. However, the functional significance of the unique role of miRNAs has yet to be elucidated in SCI. In the present study, a rat SCI model was established and a miRNA microarray analysis was performed to determine miRNA expression profiles at different times post-SCI. It was observed that SCI induces dysregulated miRNA expression in rats with SCI and miR-124 was one of the most significantly downregulated miRNAs in spinal cord tissues. Furthermore, the present results demonstrated that overexpression of miR-124 alleviates
SCI by improving functional recovery, reducing lesion volume and suppressing apoptosis. In addition, it was demonstrated that miR-124 inhibits Bax expression by directly targeting its 3'-UTR in BV-2 cells. The overexpression of Bax inhibits the protective effect of miR-124 on BV-2 cells treated with $\mathrm{H}_{2} \mathrm{O}_{2}$. Notably, the present results demonstrated that miR-124 may exert its protective effect on SCI by blocking the mitochondrial apoptotic pathway.

miR-124 is one of the most abundantly expressed miRNAs in the nervous system, including in the brain and spinal cord (42-44). Mammalian miR-124 was first detected in differentiating neurons and persists in mature neurons, suggesting that miR-124 serves key roles in neural development $(43,45)$. Previous studies demonstrated that the expression level of miR-124 was decreased in the brain and spinal cord tissues of rats following SCI, and its expression may affect the severity of SCI $(46,47)$. Consistent with these previous studies, the present 

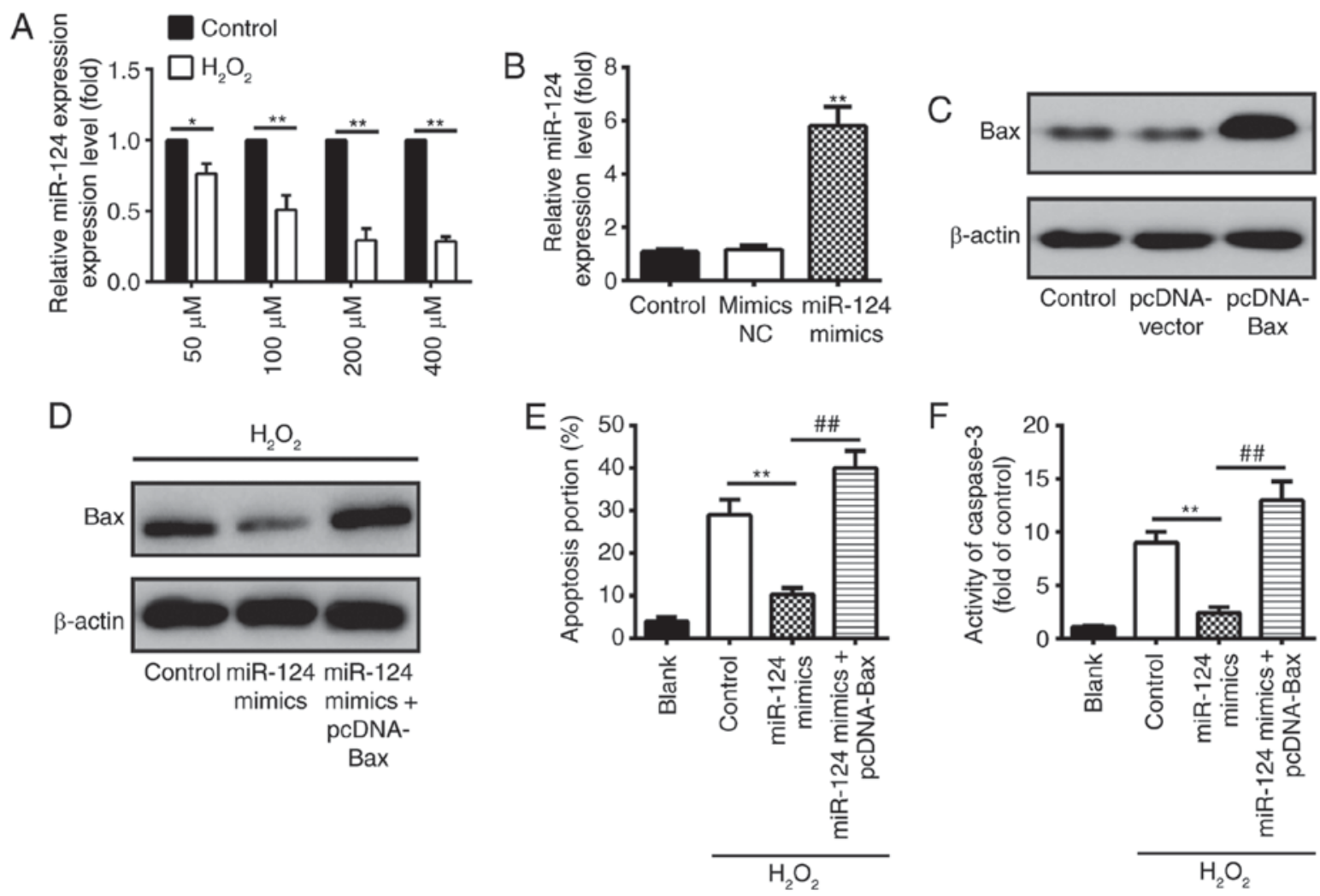

Figure 4. Restoration of Bax inhibits the protective effect of miR-124 in $\mathrm{H}_{2} \mathrm{O}_{2}$-treated BV-2 cells. (A) BV-2 cells were treated with $\mathrm{H}_{2} \mathrm{O}_{2}(50-400 \mu \mathrm{M})$ for $10 \mathrm{~h}$, and the miR-124 expression was measured using RT-qPCR analysis. ${ }^{*} \mathrm{P}<0.05,{ }^{* * *} \mathrm{P}<0.01$ vs. respective control group. (B) RT-qPCR analysis was used to measure the miR-124 expression level in BV-2 cells transfected with miR-124 mimics or mimics NC. ${ }^{* *} \mathrm{P}<0.01$ vs. mimics NC group. (C) BV-2 cells were transfected with pc-DNA-Bax or pc-DNA-vector, and Bax expression was determined by western blotting. (D) Western blotting was used to detect Bax expression in $\mathrm{H}_{2} \mathrm{O}_{2}$-treated BV-2 cells transfected with miR-124 mimics or co-transfected with miR-124 mimics and pc-DNA-Bax. Following treatment with $\mathrm{H}_{2} \mathrm{O}_{2}$, cells were transfected with miR-124 mimics or co-transfected with miR-124 mimics and pc-DNA-Bax, and (E) apoptotic cells and (F) caspase-3 activity were measured using flow cytometry and a colorimetric activity assay, respectively. ${ }^{* *} \mathrm{P}<0.01,{ }^{\# \#} \mathrm{P}<0.01$. Data are presented as the mean \pm standard deviation of three individual experiments. Bax, apoptosis regulator BAX; miR, microRNA; RT-qPCR, reverse transcription-quantitative polymerase chain reaction; $\mathrm{NC}$, negative control.
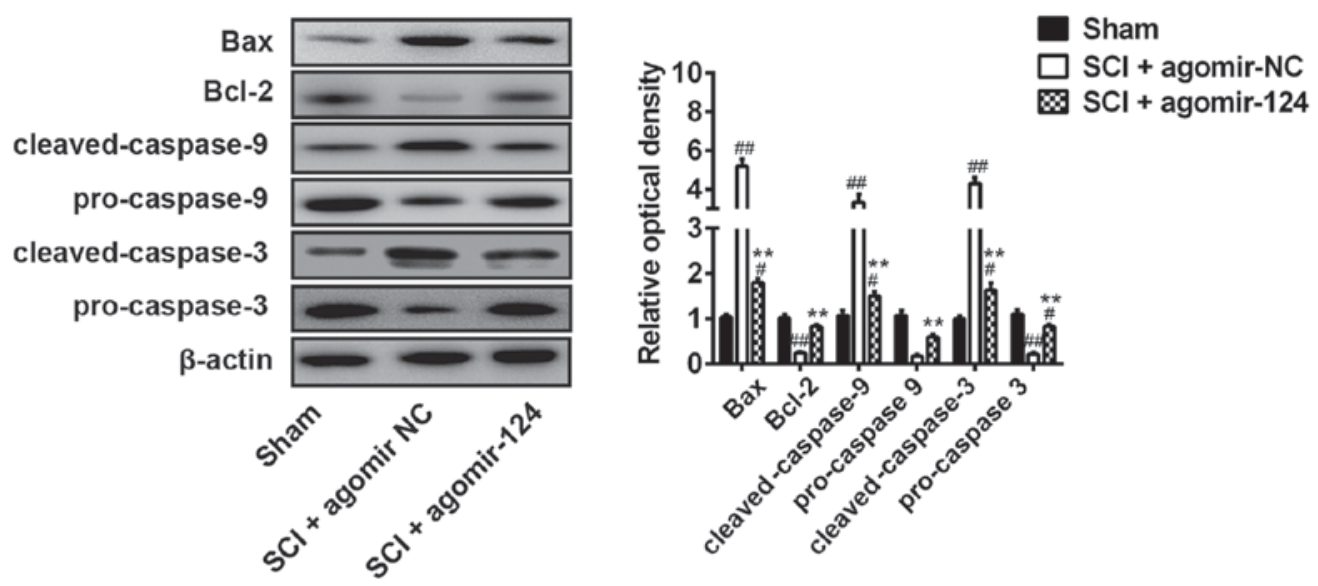

Figure 5. MicroRNA-124 blocks the mitochondrial apoptotic pathway. Rats were subjected to SCI and treated intrathecally with agomir-124 or agomir-NC, and western blot analysis was conducted to determine the Bax, Bcl-2, cleaved-caspase-9, pro-caspase-9, cleaved-caspase-3 and pro-caspase-3 expression levels in spinal cord tissues. $\beta$-actin was used as an internal control. Data are presented as the mean \pm standard deviation of three individual experiments. ${ }^{* *} \mathrm{P}<0.01$ vs. SCI + agomir-NC group; ${ }^{\#} \mathrm{P}<0.05,{ }^{* \#} \mathrm{P}<0.01$ vs. sham group. SCI, spinal cord injury; NC, negative control; Bax, apoptosis regulator BAX; Bcl-2, B-cell lymphoma-2.

results demonstrated that SCI alters miRNA expression and miR-124 was one of the most significantly downregulated miRNAs in the SCI group. A previous study reported that miR-124 exerts protective effects on SCI via regulation of neural stem cells (48). In the present study, the present results demonstrated that overexpression of miR-124 may improve functional recovery, reduce lesion volume and suppress apoptosis in rats, following SCI. These results suggested that 
miR-124 exerted a protective effect on rats with SCI and is a potential candidate target for SCI therapy. However, the molecular mechanism requires further clarification.

Mitochondria serve an important role in the apoptotic process by releasing apoptogenic molecules, including cytochrome $c(49,50)$. Bax has been identified to have a pro-apoptotic effect, which may open the permeability transition pore on the mitochondrial membrane to trigger the release of cytochrome $c$ from mitochondria into the cytoplasm (37). Cytochrome $c$ may trigger the caspase-9-molulated cascade amplification reaction in the mitochondrial apoptotic pathway, which in turn processes pro-caspase-3 to generate active caspase-3 (38). In a previous study, it was identified that miR-124 exhibited a protective effect against neuron apoptosis in rats with thyroid hypofunction by decreasing Bax expression (28). In the present study, it was demonstrated that miR-124 suppresses Bax expression by targeting its 3'-UTR in BV-2 cells. Furthermore, the correlation analysis demonstrated a negative correlation between Bax and miR-124 expression in the spinal cord tissues of rats with SCI, suggesting that Bax may be a target of miR-124 in vivo. Therefore, it was hypothesized that miR-124 may regulate the mitochondrial apoptotic pathway by inhibiting Bax expression in rats with SCI. The present results suggested that upregulation of miR-124 decreased the Bax, cleaved-caspase- 9 and cleaved-caspase-3 expression levels, and increased the Bcl-2, pro-caspase- 9 and pro-caspase- 3 expression levels in the spinal cord tissues of rats with SCI. Collectively, these results suggested that miR-124 may exert its therapeutic effects on SCI by blocking the mitochondrial apoptotic pathway.

However, there are some limitations in the present study. For example, only miR-124 was explored, whereas other miRNAs may also be relevant for the pathogenesis of SCI. Additionally, the number of experimental animals was limited. In the future, further systematic and in-depth studies investigating the pathogenesis of SCI will be conducted.

In conclusion, the present results demonstrated that SCI induces miRNA aberrant expression in a rat SCI model and miR-124 was one of the most significantly downregulated miRNAs in spinal cord tissues. In addition, it was observed that overexpression of miR-124 is able to improve functional recovery, reduce lesion volume and suppress neuronal cell apoptosis in rats following SCI. Notably, the present results demonstrated that miR-124 is able to target Bax in BV-2 cells and may exert its protective effect on SCI by blocking the mitochondrial apoptotic pathway, suggesting that miR-124 may serve as a promising novel therapeutic target for the treatment of SCI.

\section{Acknowledgements}

Not applicable.

\section{Funding}

The present study was supported by the Natural Science Foundation of Shandong Province (grant no. ZR2015YL034).

\section{Availability of data and materials}

All data generated or analyzed during this study are included in this published article.

\section{Authors' contributions}

YZ conceived the study and provided experimental materials. $\mathrm{ZX}$ and $\mathrm{KZ}$ performed the experiments and wrote the paper. ZX, KZ, QW and YZ analyzed the data. All authors read and approved the final version of the manuscript.

\section{Ethics approval and consent to participate}

All experimental procedures were approved by the Animal Care and Use Committee of Shandong University (Jinan, China).

\section{Patient consent for publication}

Not applicable.

\section{Competing interests}

The authors declare that they have no competing interests.

\section{References}

1. Chen Y, Cao S, Xu P, Han W, Shan T, Pan J, Lin W, Chen X and Wang X: Changes in the expression of miR-34a and its target genes following spinal cord injury in rats. Med Sci Monit 22: 3981-3993, 2016

2. Hu W, Wang H, Liu Z, Liu Y, Wang R, Luo X and Huang Y: Neuroprotective effects of lycopene in spinal cord injury in rats via antioxidative and anti-apoptotic pathway. Neurosci Lett 642: 107-112, 2017.

3. Thuret S, Moon LD and Gage FH: Therapeutic interventions after spinal cord injury. Nat Rev Neurosci 7: 628-643, 2006.

4. Blight AR: Miracles and molecules-progress in spinal cord repair. Nat Neurosci 5 (Suppl 1): S1051-S1054, 2002.

5. Rabchevsky AG, Patel SP and Springer JE: Pharmacological interventions for spinal cord injury: Where do we stand? How might we step forward? Pharmacol Ther 132: 15-29, 2011.

6. Kawabata H, Setoguchi T, Yone K, Souda M, Yoshida H, Kawahara K, Maruyama I and Komiya S: High mobility group box 1 is upregulated after spinal cord injury and is associated with neuronal cell apoptosis. Spine (Phila Pa 1976) 35: 1109-1115, 2010.

7. Li J, Huang C-Y, Zheng RL, Cui KR and Li JF: Hydrogen peroxide induces apoptosis in human hepatoma cells and alters cell redox status. Cell Biol Int 24: 9-23, 2000

8. Reuter S, Eifes S, Dicato M, Aggarwal BB and Diederich M: Modulation of anti-apoptotic and survival pathways by curcumin as a strategy to induce apoptosis in cancer cells. Biochem Pharmacol 76: 1340-1351, 2008.

9. Balaban RS, Nemoto S and Finkel T: Mitochondria, oxidants, and aging. Cell 120: 483-495, 2005.

10. Li P, Nijhawan D and Wang X: Mitochondrial activation of apoptosis. Cell 116 (2 Suppl): S57-S61, 2004.

11. Nicholson DW and Thornberry NA: Caspases: Killer proteases. Trends Biochem Sci 22: 299-306, 1997.

12. Croce CM: Causes and consequences of microRNA dysregulation in cancer. Nat Rev Genet 10: 704-714, 2009.

13. Bartel DP: MicroRNAs: Target recognition and regulatory functions. Cell 136: 215-233, 2009.

14. Friedman RC, Farh KK, Burge CB and Bartel DP: Most mammalian mRNAs are conserved targets of microRNAs. Genome Res 19: 92-105, 2009.

15. Krichevsky AM: MicroRNA profiling: From dark matter to white matter, or identifying new players in neurobiology. ScientificWorldJournal 7: 155-166, 2007.

16. Kosik KS: The neuronal microRNA system. Nat Rev Neurosci 7: 911-920, 2006.

17. Bak M, Silahtaroglu A, Møller M, Christensen M, Rath MF, Skryabin B, Tommerup N and Kauppinen S: MicroRNA expression in the adult mouse central nervous system. RNA 14: 432-444, 2008 
18. Liu NK, Wang XF, Lu QB and Xu XM: Altered microRNA expression following traumatic spinal cord injury. Exp Neurol 219: 424-429, 2009

19. Liu G, Keeler BE, Zhukareva V and Houlé JD: Cycling exercise affects the expression of apoptosis-associated microRNAs after spinal cord injury in rats. Exp Neurol 226: 200-206, 2010.

20. Sun Y, Su Q, Li L, Wang X, Lu Y and Liang J: MiR-486 regulates cardiomyocyte apoptosis by p53-mediated BCL-2 associated mitochondrial apoptotic pathway. BMC Cardiovasc Disord 17: 119, 2017.

21. Makhdoumi P, Roohbakhsh A and Karimi G: MicroRNAs regulate mitochondrial apoptotic pathway in myocardial ischemia-reperfusion-injury. Biomed Pharmacother 84: 1635-1644, 2016.

22. Wang J, Jiao Y, Cui L and Jiang L: miR-30 functions as an oncomiR in gastric cancer cells through regulation of P53-mediated mitochondrial apoptotic pathway. Biosci Biotechnol Biochem 81: 119-126, 2017.

23. Yune TY, Lee JY, Jung GY, Kim SJ, Jiang MH, Kim YC, $\mathrm{Oh}$ YJ, Markelonis GJ and Oh TH: Minocycline alleviates death of oligodendrocytes by inhibiting pro-nerve growth factor production in microglia after spinal cord injury. J Neurosci 27: 7751-7761, 2007

24. Saeed AI, Sharov V, White J, Li J, Liang W, Bhagabati N, Braisted J, Klapa M, Currier T, Thiagarajan M, et al: TM4: A free, open-source system for microarray data management and analysis. Biotechniques 34: 374-378, 2003.

25. Livak KJ and Schmittgen TD: Analysis of relative gene expression data using real-time quantitative PCR and the 2(-Delta Delta C(T)) method. Methods 25: 402-408, 2001

26. Basso DM, Beattie MS and Bresnahan JC: A sensitive and reliable locomotor rating scale for open field testing in rats J Neurotrauma 12: 1-21, 1995.

27. Yu JY, Chung KH, Deo M, Thompson RC and Turner DL: MicroRNA miR-124 regulates neurite outgrowth during neuronal differentiation. Exp Cell Res 314: 2618-2633, 2008.

28. Shao Q, Jiang W and Jin Y: MiR-124 effect in neurons apoptosis in newborn rat with thyroid hypofunction. Int J Clin Exp Pathol 8: 14465-14471, 2015.

29. Yu DS, Lv G, Mei XF, Cao Y, Wang YF, Wang YS and Bi YL: MiR-200c regulates ROS-induced apoptosis in murine BV-2 cells by targeting FAP-1. Spinal Cord: Dec 2, 2014 (Epub ahead of print).

30. Hu F, Min J, Cao X, Liu L, Ge Z, Hu J and Li X: MiR-363-3p inhibits the epithelial-to-mesenchymal transition and suppresses metastasis in colorectal cancer by targeting Sox4. Biochem Biophys Res Commun 474: 35-42, 2016.

31. Nishimura R, Tabata K, Arakawa M, Ito Y, Kimura Y, Akihisa T, Nagai H, Sakuma A, Kohno H and Suzuki T: Isobavachalcone, a chalcone constituent of Angelica keiskei, induces apoptosis in neuroblastoma. Biol Pharm Bull 30: 1878-1883, 2007.

32. He J, Xiao Y, Casiano CA and Zhang L: Role of mitochondrial cytochrome $c$ in cocaine-induced apoptosis in coronary artery endothelial cells. J Pharmacol Experimental Ther 295: 896-903, 2000.

33. Liu H, Qin CK, Han GQ, Xu HW, Ren WH and Qin CY: Synthetic chenodeoxycholic acid derivative, HS-1200, induces apoptosis of human hepatoma cells via a mitochondrial pathway. Cancer Lett 270: 242-249, 2008.

34. Noguchi K, Kitanaka C, Yamana H, Kokubu A, Mochizuki T and Kuchino Y: Regulation of c-Myc through phosphorylation at Ser-62 and Ser-71 by c-Jun N-terminal kinase. J Biol Chem 274 32580-32587, 1999.

35. Klapsinou E, Argyri E, Panotopoulou E, Daskalopoulou D, Patsouris E, Nonni A, Lazaris AC and Thomopoulou GH: Bax and Bak expression in cervical smears of women with low-and high-risk HPV types: A study of 120 cases. J Cytol 32: 223-229, 2015 .
36. Su CC, Lee KI, Chen MK, Kuo CY, Tang CH and Liu SH: Cantharidin induced oral squamous cell carcinoma cell apoptosis via the JNK-regulated mitochondria and endoplasmic reticulum stress-related signaling pathways. PLoS One 11: e0168095, 2016.

37. Lu Z, Chen H, Zheng XM and Chen ML: Experimental study on the apoptosis of cervical cancer Hela cells induced by juglone through c-Jun N-terminal kinase/c-Jun pathway. Asian Pac J Trop Med 10: 572-575, 2017.

38. Zou H, Li Y, Liu X and Wang X: An APAF-1.cytochrome $c$ multimeric complex is a functional apoptosome that activates procaspase-9. J Biol Chem 274: 11549-11556, 1999.

39. Giovanni SD, Knoblach SM, Brandoli C, Aden SA, Hoffman EP and Faden AI: Gene profiling in spinal cord injury shows role of cell cycle neuronal death. Ann Neurol 53: 454-468, 2003

40. De Biase A, Knoblach SM, Di Giovanni S, Fan C, Molon A, Hoffman EP and Faden AI: Gene expression profiling of experimental traumatic spinal cord injury as a function of distance from impact site and injury severity. Physiol Genomics 22: 368-381,2005.

41. Hu JZ, Huang JH, Zeng L, Wang G, Cao M and Lu HB: Anti-apoptotic effect of microRNA-21 after contusion spinal cord injury in rats. J Neurotrauma 30: 1349-1360, 2013.

42. Lagos-Quintana M, Rauhut R, Yalcin A, Meyer J, Lendeckel W and Tuschl T: Identification of tissue-specific MicroRNAs from mouse. Curr Biol 12: 735-739, 2002.

43. Krichevsky AM, King KS, Donahue CP, Khrapko K and Kosik KS: A microRNA array reveals extensive regulation of microRNAs during brain development. RNA 9: 1274-1281, 2003.

44. Sempere LF, Freemantle S, Pitha-Rowe I, Moss E, Dmitrovsky E and Ambros V: Expression profiling of mammalian microRNAs uncovers a subset of brain-expressed microRNAs with possible roles in murine and human neuronal differentiation. Genome Biol 5: R13, 2004.

45. Miska EA, Alvarez-Saavedra E, Townsend M, Yoshii A Sestan N, Rakic P, Constantine-Paton M and Horvitz HR: Microarray analysis of microRNA expression in the developing mammalian brain. Genome Biol 5: R68, 2004.

46. Zhao Y, Zhang H, Zhang D, Yu CY, Zhao XH, Liu FF, Bian GL, Ju $\mathrm{G}$ and Wang J: Loss of microRNA-124 expression in neurons in the peri-lesion area in mice with spinal cord injury. Neural Regen Res 10: 1147-1152, 2015

47. Song JL, Zheng W, Chen W, Qian Y, Ouyang YM and Fan CY: Lentivirus-mediated microRNA-124 gene-modified bone marrow mesenchymal stem cell transplantation promotes the repair of spinal cord injury in rats. Exp Mol Med 49: e332, 2017.

48. Xu W, Wang X, Li P, Qin K and Jiang X: miR-124 regulates neural stem cells in the treatment of spinal cord injury. Neurosci Lett 529: 12-17, 2012.

49. Malhotra R, Lin Z, Vincenz C and Brosius FC III: Hypoxia induces apoptosis via two independent pathways in Jurkat cells: Differential regulation by glucose. Am J Physiol Cell Physiol 281: C1596-C1603, 2001

50. Susin SA, Lorenzo HK, Zamzami N, Marzo I, Snow BE Brothers GM, Mangion J, Jacotot E, Costantini P, Loeffler M, et al: Molecular characterization of mitochondrial apoptosis-inducing factor. Nature 397: 441-446, 1999.

This work is licensed under a Creative Commons Attribution 4.0 International (CC BY-NC 4.0) License 